\title{
Characterization and staging of rectal tumors: endoscopic ultrasound versus MRI / CT. Pictorial essay.
}

\author{
Radu Badea1, Mariana M. Gersak², Sorin M. Dudea², Florin Graur ${ }^{3}$, Nadim Al Hajjar³, \\ Luminița Furcea ${ }^{3}$
}

${ }^{1}$ Ultrasound Imaging Laboratory, Department of Imaging and Radiology, "Octavian Fodor" Regional Institute of Gastroenterology and Hepatology, "Iuliu Hatieganu" University of Medicine and Pharmacy ${ }^{2}$ Department of Radiology Cluj County Clinical Emergency Hospital, "Iuliu Hatieganu” University of Medicine and Pharmacy, ${ }^{3} 3^{\text {rd }}$ Surgical Clinic, "Octavian Fodor” Regional Institute of Gastroenterology and Hepatology Cluj-Napoca, Romania

\begin{abstract}
Endoscopic ultrasound is recommended for rectal cancer staging. Transrectal ultrasound approach is able to overcome one of the limitations of this technique regarding circumferential rectal stenosis. Prior intrarectal administration of a small amount of fluid contrast agent optimizes the method, making it easier to distinguish the layers of the rectal wall and tumor formation. Endoscopic ultrasound focuses on the gray-scale mode. Additional procedures provide useful information for tumor assessment: Doppler ultrasound helps identify chaotic intratumoral vascularization; 3D ultrasound allows the assessment and accurate measurement of the tumor; elastography can identify focal tumor dysplasia within adenomas; contrast-enhanced ultrasound allows characterization of tumor microvasculature. Even if they are not as accurate in distinguishing rectal wall layers, cross-sectional imaging techniques (CT, MRI) can identify the anatomical relationships of advanced locoregional cancers, as well as possible distant metastasis. This paper aims at illustrating the main pathological aspects of endoscopic ultrasound multimodal examination useful for cancer staging.
\end{abstract}

Keywords: endoscopic ultrasound, CEUS, color elastography, rectal tumors

\section{Introduction}

The diagnosis, treatment and follow-up of patients with colorectal cancer has considerably improved in the recent years. Given the tendency to choose the optimal and at the same time least invasive treatment, the role of imaging methods have gained in importance for the diagnosis, staging, and follow-up of patients with colorectal cancer. Thorough rectal cancer assessment and staging includes the local examination of the tumor (indicating the extent of rectal wall and neighboring tissues invasion

Received 28.04.2015 Accepted 10.05.2015

Med Ultrason

2015, Vol. 17, No 2, 241-247

Corresponding author: Mariana M. Gersak

Radiology Clinic

Cluj County Emergency Clinical Hospital

3-5 Clinicilor Street

400006, Cluj-Napoca, Romania

E-mail:mariana.gersak@umfcluj.ro and the assessment of adjacent lymph nodes [1], the identification of any distant metastases and synchronous intestinal tumors [2]. There are several imaging techniques that can be used for rectal cancer assessment: endoscopic ultrasound, which is the most accessible and with good results for cancer staging; computed tomography (CT) and magnetic resonance imaging (MRI) are cross-sectional investigations that scan the entire body, identifying possible distant metastases or synchronous tumors. Among these, endoscopic ultrasound has proven to be the best imaging technique in rectal tumors. A complete endoscopic ultrasound multimodal examination may even surpass biopsy in terms of rectal cancer detection $[3,4]$

\section{Ultrasound techniques}

2D and 3D gray scale ultrasound. Endorectal and endovaginal US, in one term endoscopic US, represent the essential test for the local assessment of rectal tu- 
mors. It is easy to perform and well-tolerated, requiring minimal prior preparation (enema), providing essential morphological information for cancer diagnosis and staging (uTNM). When performed after administration of small quantities of water $(100-150 \mathrm{cc})$, the method can be a very useful diagnostic tool (fig 1). Transrectal (TR) US is useful for the assessment of tumor morphology and tumor size, tumor extension into the longitudinal rectal muscle and inside it, tumor location in relation to the anus, and perirectal lymph node characterization [5,6]. All these aspects are summarized in Table I [7]. 3D US allows accurate measurement of rectal tumors and identification of anatomical relationships, which assists the surgical planning procedure. Processing the data acquired by 3D US makes possible the visualization of rectal tumors in a way similar to digestive endoscopy (fig 2).

Elastography. It is an ultrasound application that has already proved useful in various clinical fields [8]. So far, the association between TRUS and elastography proved particularly useful in assessing prostate, rectal tumors being less studied. Of all types of elastography (ARFI, SWE and color/strain elastography), rectal cancer assessment was mainly based on strain/color elastography (SE), probably because this type of elastography is commonly present with ultrasound equipment (fig 3).

Doppler ultrasound. This technique is useful for the assessment of rectal tumors, providing information about the characteristics of the blood vessels located in the intra- and peritumoral areas. Color flow mapping (CFM) identifies the presence of blood vessels and the direction of the blood flow, while the spectral method provides information on the characteristics, direction and the type of blood flow.

Contrast-enhanced ultrasound (CEUS). This application of TRUS has not yet been fully explored in relation to rectal tumors, but there are many studies in other areas

Table I. Rectal cancer staging [7].

\begin{tabular}{|c|c|}
\hline $\begin{array}{l}\mathrm{T} \\
\text { primary tumor }\end{array}$ & $\begin{array}{l}\text { Tx - primary tumor cannot be assessed } \\
\text { T0 - no evidence of primary tumor } \\
\text { Tis - carcinoma in situ: intraepithelial tumor or invasion of lamina propria } \\
\text { T1 - tumor invades submucosa } \\
\text { T2 - tumor invades muscularis propria } \\
\text { T3 - tumor invades through the muscularis propria into pericolorectal tissues } \\
\text { T4a - tumor penetrates to the surface of the visceral peritoneum } \\
\text { T4b - tumor directly invades or is adherent to other organs or structures. }\end{array}$ \\
\hline $\begin{array}{l}\mathrm{N} \\
\text { regional lymph nodes }\end{array}$ & $\begin{array}{l}\mathrm{Nx} \text { - regional lymph nodes cannot be assessed } \\
\mathrm{N} 0 \text { - no regional lymph node metastasis } \\
\mathrm{N} 1-\text { metastases in } 1-3 \text { regional lymph nodes } \\
\mathrm{N} 1 \mathrm{a}-\text { metastasis in } 1 \text { regional lymph node } \\
\mathrm{N} 1 \mathrm{~b} \text { - Metastases in } 2-3 \text { regional lymph nodes } \\
\mathrm{N} 1 \mathrm{c} \text { - tumor deposit(s) in the subserosa, mesentery or nonperitonealized pericolic, or perirectal tissues } \\
\text { without regional nodal metastasis } \\
\mathrm{N} 2-\text { metastases in } \geq 4 \text { regional lymph nodes } \\
\mathrm{N} 2 \mathrm{a}-\text { metastases in } 4-6 \text { regional lymph nodes } \\
\mathrm{N} 2 \mathrm{~b}-\text { Metastases in } \geq 7 \text { regional lymph nodes }\end{array}$ \\
\hline $\begin{array}{l}\mathrm{M} \\
\text { distant metastasis }\end{array}$ & $\begin{array}{l}\text { M0 - no distant metastasis } \\
\text { M1 - distant metastasis } \\
\text { M1a - metastasis confined to } 1 \text { organ or site (e.g. liver, lung, ovary, non-regional node). } \\
\text { M1b - metastases in }>1 \text { organ/site or the peritoneum }\end{array}$ \\
\hline
\end{tabular}

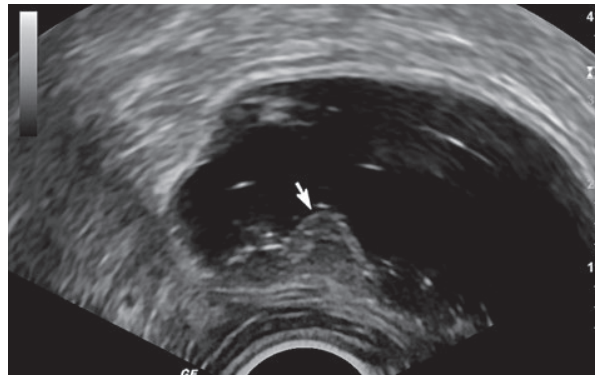

Fig 1. Transvaginal ultrasound with rectal relaxation using water enema. Stage T2 villous tumor (arrow).

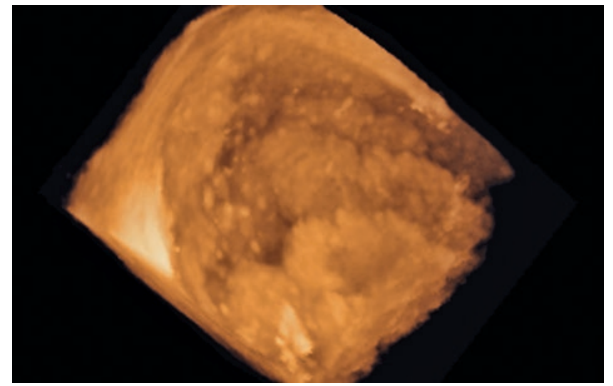

Fig 2. Transrectal ultrasound - three-dimensional reconstruction of villous adenoma. 


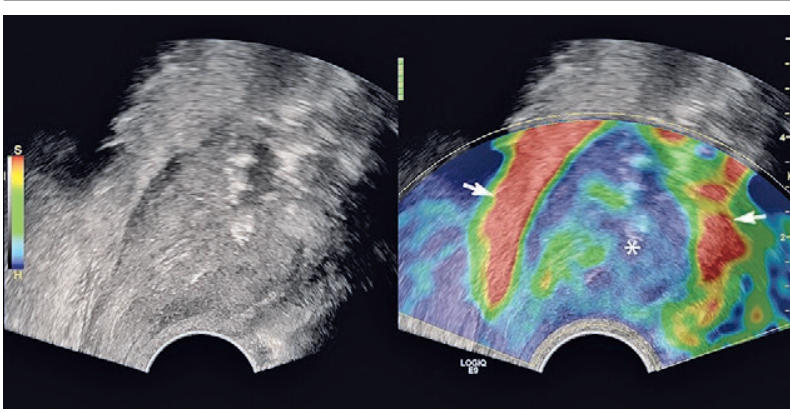

Fig 3. Color elastography. Rigid tumor formation (*) as compared to perirectal soft tissues (arrows).

of interest, where it has demonstrated its importance in the characterization of tumoral lesions. Immediately after the injection of the contrast medium, the microbubbles reach the rectum, especially the areas where microcirculation is more abundant. The images must be stored for further analysis of loading and unloading curves and for a more objective interpretation, according to the time intervals acquired on these curves. In order to calculate these curves and easily identify the differences between them, one or more ROIs must be manually selected on the equipment, one for the tumor-bearing region and another for an apparently tumor-free region. The most important time-intensity parameters that can be acquired on the loading and unloading curves are the following: contrast arrival time in the ROI (AT), maximum contrast medium enhancement (time to peak - TTP), maximum contrast intensity (peak intesity - PI), area under the curve (AUC), loading time (WIT), ascending slope (AS) and echo intensity (EI) [9-11]. With the help of these parameters, CEUS offers very good interobserver variability [9].

\section{Cross-sectional imaging techniques}

CT. It is a radiating technique, but it has the advantage of rapid acquisition scans. Preparation prior to CT scan includes water enemas. Portal-phase CT scan was proposed in order to reduce the degree of radiation, with similar results obtained for arterial and venous phases [12]. CT with intravenous administration of iodine based contrast medium shows limited soft tissue contrast for local staging of early tumors [2,5]. CT scan cannot distinguish between the layers of the rectal wall [13]. However, multidetector CT (MDCT) with multiplanar reconstruction (MPR) has a good accuracy for the assessment of locoregionally advanced tumors (T3, T4) $(87.1 \%$ for tumor assessment and $84.8 \%$ for lymph node assessment) [13].

MRI. It is a non-radiating cross-sectional imaging technique, but the acquisition of all sequences takes longer than in the case of a CT scan. However, MRI has be- come increasingly popular for rectal cancer diagnosis and staging. Compared to CT, MRI shows better soft tissue contrast and, in addition, it has many advantages: it emphasizes tumor relationships with the rectum, mesorectal fascia, and adjacent tissues, it identifies pelvic lymph nodes and possible vascular infiltration [2,14]. The use of the endorectal coil increases the diagnostic value of the method in the evaluation of rectal tumors. The basic MRI protocol includes either the $\mathrm{T} 2$ sequence alone, or the additional T1 sequence. Using fat-suppressed T2 sequences is useful to emphasize perirectal fat invasion [15]. The administration of Gadolinium based contrast agents does not bring additional information for the diagnostic accuracy of magnetic resonance imaging. Of all MRI sequences, the $\mathrm{T} 2$ sequence is the most appropriate to identify rectal wall layers. This sequence is characterized by hyperintense mucosa and submucosa, which makes it difficult to distinguish one from the other; relatively hypointense muscularis propria; hyperintense layer representing the perirectal fat tissue. The mesorectal fascia is a linear structure, smooth and hypointense, encompassing the mesorectum [15] (fig 4).

Endoscopic US assessment of rectal cancer. Rectal tumors present varied echogenicity, and in evolution determine disruption and upheaval of rectal layers. TRUS allows identification of tumor morphology, size and loca-

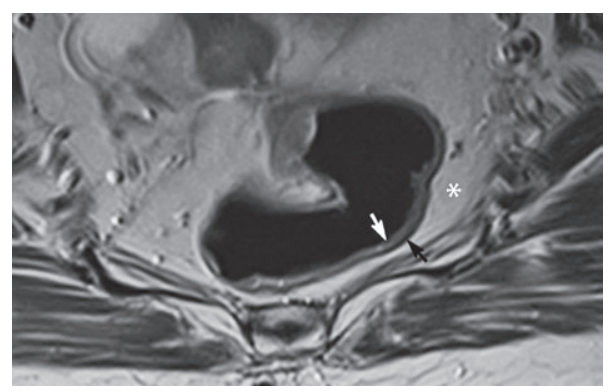

Fig 4. MRI scan without contrast medium, coronal section. Normal stratification of the rectum: mucosa and submucosa (white arrow); muscle (black arrow); perirectal tissue $(*)$.

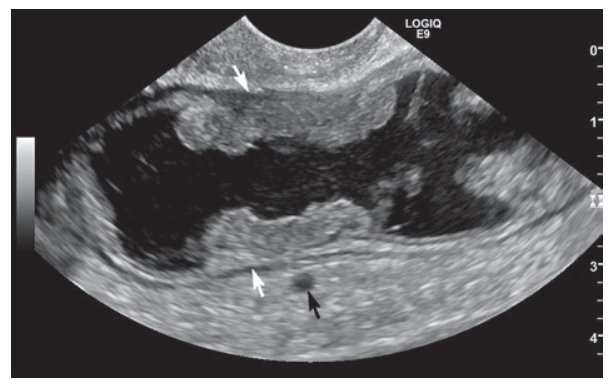

Fig 5. Hypoechoic rectal tumor, circumferential, stage T3 (white arrows). Small peritumoral lymph node (black arrow). 


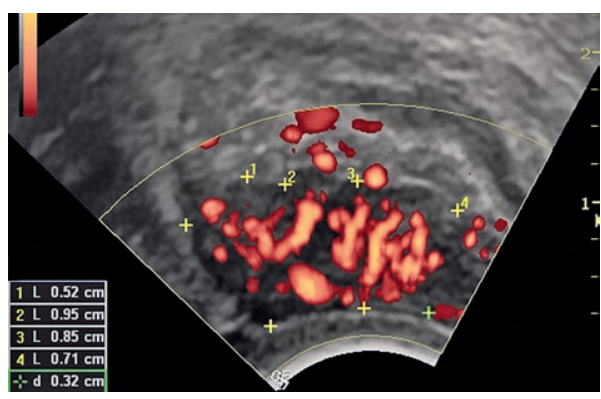

Fig 6. Power Doppler ultrasound. Rectal tumor with intense and chaotic vascularization (stage T2).

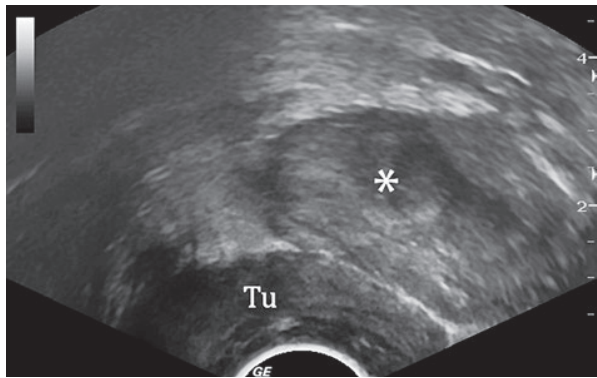

Fig 7. Hypoechoic rectal tumor, circumferential $(\mathrm{Tu})$, which comes in contact with the prostate gland $(*)$, stage T4.

tion in relation to the anus, assessment of lymph node morphology and size, if present, and, of course, uTNM staging [5] (fig 5-7). 3D US shows similar or even higher accuracy than 2D US in the evaluation of rectal tumors (Acc $=78-88 \%$ for $3 \mathrm{D}$ and $69-84 \%$ for $2 \mathrm{D}$ US) $[16,17]$. The 3D image can be impressive.

Pathological lymph nodes with $\geq 5-7 \mathrm{~mm}$ in size are hypoechoic and round [5,18-20].

Rectal tumors have intense and chaotic vasculature (fig 8). The contribution of Doppler ultrasound to cancer staging is not significant, but it is extremely useful to distinguish tumor recurrence from postoperative scarring [21]. Malignant lymph nodes have a $\mathrm{RI} \geq 0.61$ and $\mathrm{PSV} \geq 20 \mathrm{~cm} / \mathrm{second}[20]$.

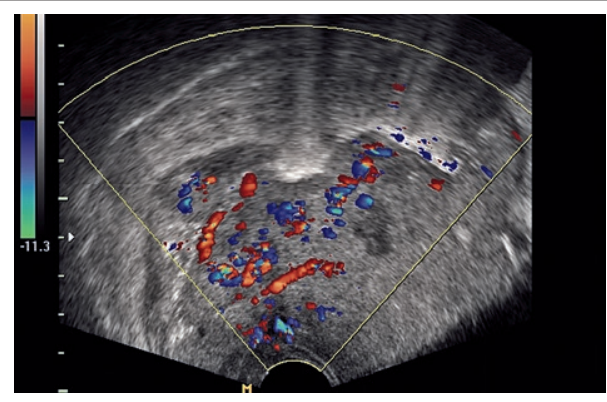

Fig 8. Color Doppler ultrasound of a large hypoechoic rectal tumor with intense vascularization and irregular vascular distribution.

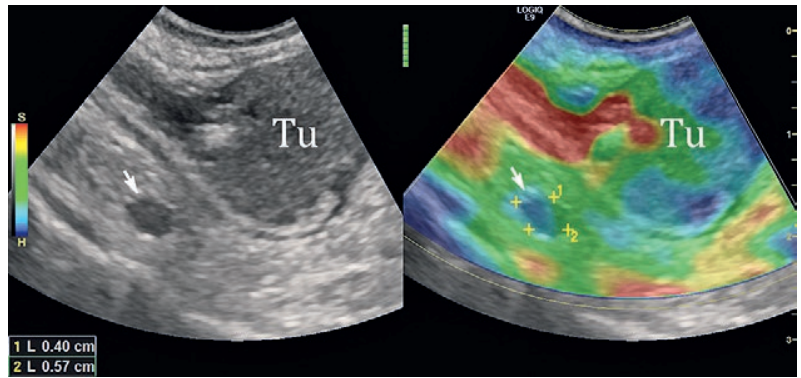

Fig 9. Rigid lymph node for color elastography (arrow). Tu = tumor

Elastography can distinguish malignant form benign tumors (malignant tumor mean strain ratio value over 1.25 and benign tumor mean strain ratio value below 1.25 [4]. In terms of cancer staging, elastography, along with TRUS, is useful to distinguish pT0 stage from $\mathrm{pT} 1$ stage, with better results than MRI, including pretreatment biopsy. Elastography, additional to TRUS, does not bring additional information regarding T2 and T3 staging, as TRUS is sufficient for final cancer staging [3] (fig 9). To our knowledge, the characterization of pathological lymph nodes did not include their elasticity but, by our experience, these appear more rigid than perirectal fat during elastography (fig 10).

CEES. The contrast agent is homogeneously retained by all rectal adenomas, and, compared to tumor-

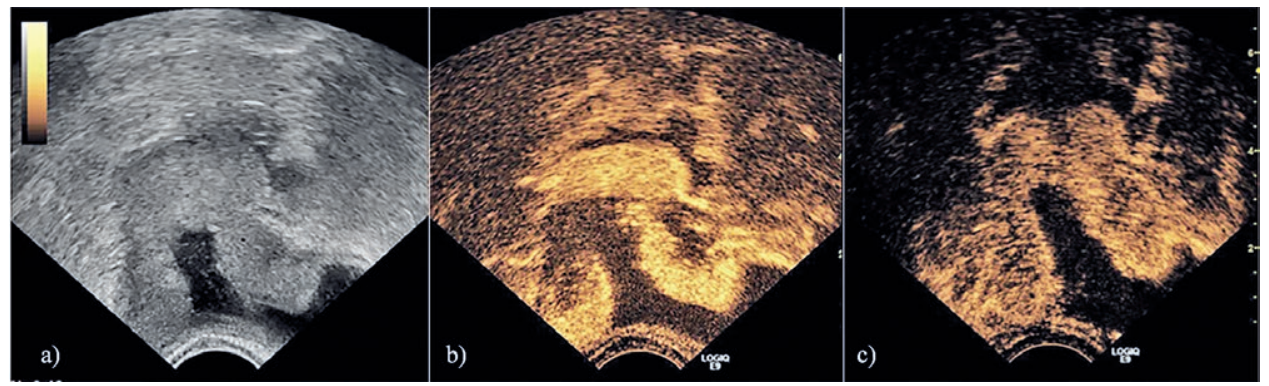

Fig 10. a) Circumferential rectal tumor gray scale; CEUS: b) Rapid and intense uptake in the arterial phase $(19 \mathrm{sec})$; c) Rapid contrast washout in the venous phase $(46 \mathrm{sec})$. 


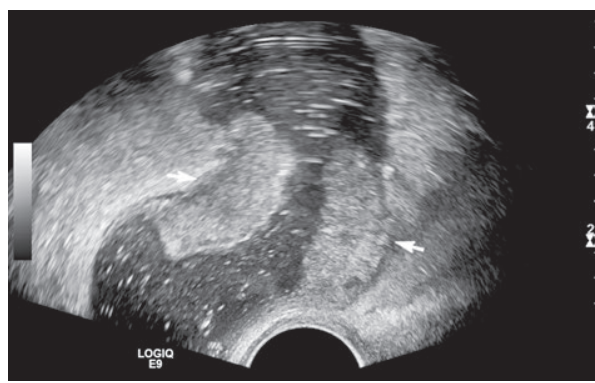

Fig 11. Transvaginal ultrasound. Hypoechoic circumferential rectal tumor, stage T3 (arrows).

free rectal wall the contrast medium becomes visible later and with lower intensity. Difficulty may arise in case of larger adenomas with increased uptake. Adenocarcinomas are irregular and, compared to the tumor-free rectal wall, the contrast medium loads faster and contrast maximum enhancement occurs earlier [10]. Specifically, aggressive tumors with high degree of angiogenesis have an increased inhomogeneous contrast uptake. An exception to this rule is that of large tumors with intratumoral necrosis, which can have a low contrast uptake or even no uptake [10] (fig 11).

\section{Limitations}

There are relatively few limitations of endoscopic US; difficulties may occur in the case of high rectal tumors, where the transducer cannot penetrate, in the evaluation of circumferential neoplasms, stenotic in men, in case of posterior rectal curving, which may limit optimal visualization of tumors on the posterior wall of the rectum. Since the introduction of the water enema procedure, the presence of air and feces in the rectum is no longer a significant drawback [22-24]. However, there is still the question whether the presence of water does not restrict the use of elastography, giving rise to artifacts. Also, the transvaginal approach can be used for stenotic tumors, circumferential in women, thus offering a much better visualization than the transrectal approach $[22,25]$.

CT. Even if CT cannot differentiate rectal wall layers, rectal tumors can be identified using iodine-containing contrast medium, involving the perirectal fat tissue and adjacent organs (in more advanced stages). To assess T3T4 tumor stages, CT with MPR showed good accuracy (87.1\%) [13] (fig12, fig 13). CT scan is inferior to TRUS and MRI with endorectal coil when identifying peritumoral lymph node metastasis [26].

MRI. Rectal lesions show increased signal intensity than the muscle layer and lower signal intensity than the submucosa [27]. Identification of tumor lymph nodes assessed using MRI strictly from a size perspective is not plausible [28]. To identify lymph node invasion using MRI, lymph node morphology must be taken into account, such as the irregular contours and inhomogeneous intranodal signal intensity, rather than their size [28]. In

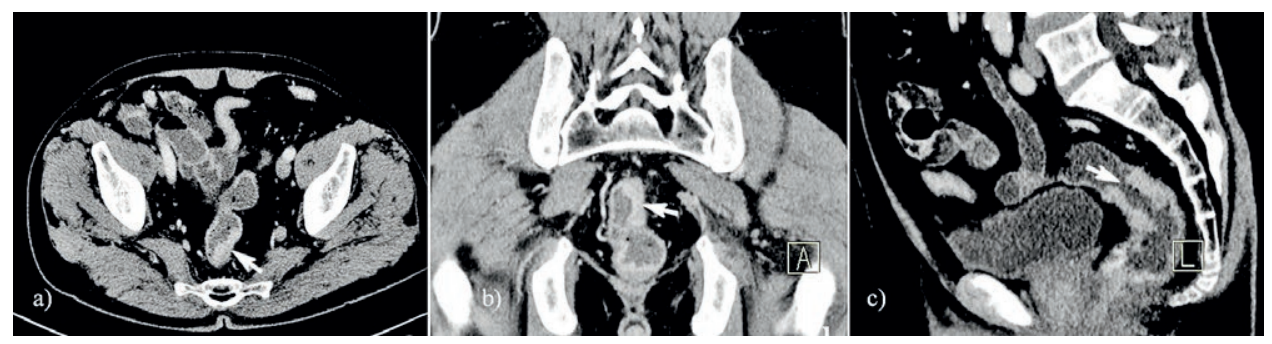

Fig 12. CT scan- axial (a), coronal (b) and sagittal view (c), with iv. contrast medium. Circumferential rectal tumor, with contrast uptake - stage T3 (arrows). Courtesy of Dr. Adrian Brumboiu.

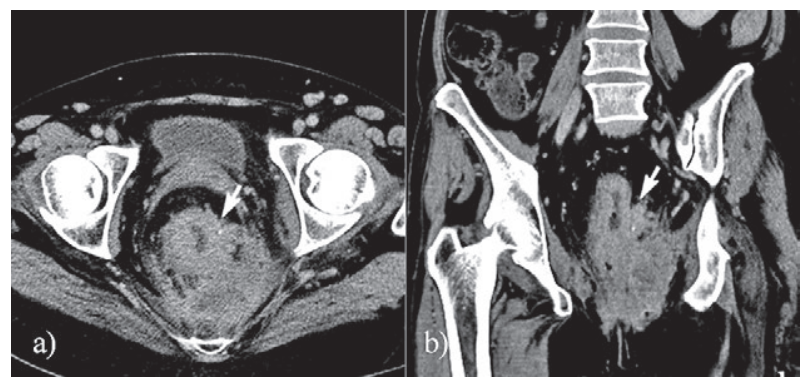

Fig 13. CT scan - axial (a) and coronal (b) view, with iv. contrast medium. Rectal tumor with contast uptake and perineal extension - stage T4 (arrows). Courtesy of Dr. Adrian Brumboiu. 


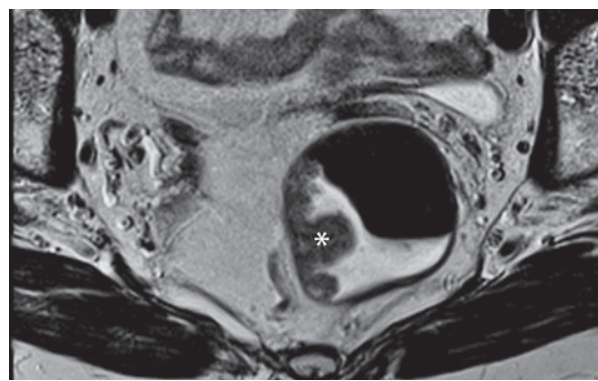

Fig 14. MRI scan - bulging rectal tumor formation, confined to the muscular layer - stage $\mathrm{T} 2(*)$

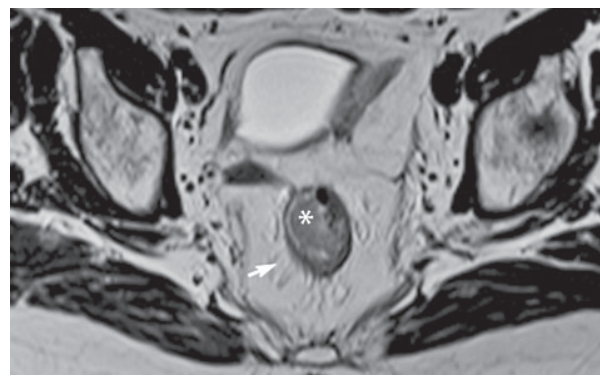

Fig 15. MRI scan - rectal tumor formation, stage T3 (*) with perirectal fat tissue invasion (arrow).

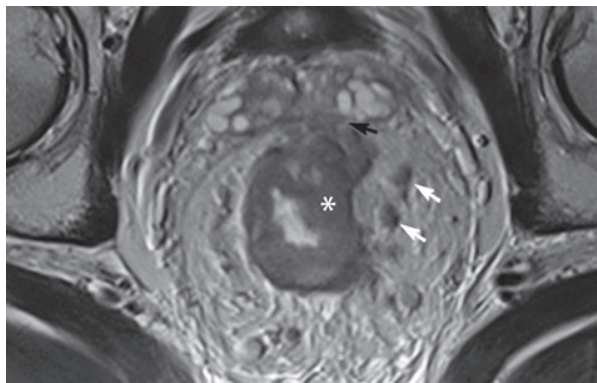

Fig 16. MRI scan - circumferential rectal tumor formation, stage T4 (*), with seminal vesicle invasion (black arrow) and lymph nodes (white arrows).

evaluating perirectal lymph nodes, MRI was significantly improved by using the endorectal coil, obtaining the highest accuracy of all imaging investigations [26] (fig 14-16).

The main limitation of CT is that it cannot identify the rectal layers individually and it shows a low soft tissue contrast, thus unable to identify early stage rectal cancer. It should also be taken into account that this type of examination cannot be performed in people allergic to iodine. Regarding MRI, some of its drawbacks include the high costs, poor accessibility, the length of the procedure and general contraindications in patients with pacemakers, various metal prostheses and in those who suffer from claustrophobia.

\section{Conclusions}

Gray scale endoscopic US is a good choice for the local assessment of rectal tumors. Additionally, every TRUS application brings useful information in the diagnosis, staging and reassessment of rectal tumors: Doppler US emphasizes intratumoral hypervascularization and differentiates between postsurgical scarring and local recurrences; 3D US measures the volume, the exact size of the tumor, identifying its spatial position and the relationships with adjacent tissues; elastography is useful for early rectal cancer staging and for the differentiation between malignant tumors and adenomas; CEUS identifies intratumoral microcirculation. All these applications have the great advantage of being accessible and applicable as part of the same examination, radiation-free and with good diagnostic accuracy. Endoscopic US cannot replace cross-sectional examinations that can scan the entire body of the patient, in search of possible distant metastasis, but when it comes to local examination, it is the method of choice for all rectal tumors.

Conflict of interest: none

\section{References}

1. Beets-Tan RG, Beets GL. Rectal Cancer: Review with Emphasis on MR Imaging. Radiology 2004; 232: 335-346.

2. Tapan Ü, Özbayrak M, Tatli S. MRI in local staging of rectal cancer: an update. Diagn Interv Radiol 2014; 20: 390398.

3. Waage JE, Leh S, Rosler C, et al. Endorectal ultrasonography, strain elastography and MRI differentiation of rectal adenomas and adenocarcinomas. Colorectal Dis $2014 \mathrm{Nov}$ 18. doi: $10.1111 /$ codi. 12845 .

4. Waage JE, Havre RF, Odegaard S, Leh S, Eide GE, Baatrup G. Endorectal elastography in the evaluation of rectal tumours. Colorectal Dis 2011; 13: 1130-1137.

5. Akbari RP, Wong WD. Endorectal ultrasound and the preoperative staging of rectal cancer Scand J Surg 2003; 92: 25-33.

6. Hildebrandt U, Feifel G. Preoperative staging of rectal cancer by intrarectal ultrasound. Dis Colon Rectum 1985; 28 : 42-46.

7. How is colorectal cancer staged? Available at: http://www. cancer.org/cancer/colonandrectumcancer/detailedguide/ colorectal-cancer-staged. Accesed 2015 May 5.

8. Cosgrove D, Piscaglia F, Bamber J, et al. EFSUMB Guidelines and recommendations on the clinical use of ultrasound elastography. Part 2: Clinical applications. Ultraschall Med 2013; 34: 238-253.

9. Zhuang H, Yang ZG, Wang ZQ, et al. Features of timeintensity curve parameters of colorectal adenocarcinomas 
evaluated by double-contrast enhanced ultrasonography: initial observation. Eur J Radiol 2012; 81: 677-682.

10. Lu M, Yan B, Song J, Ping W, Yue LX, Song B. Doublecontrast-enhanced sonography for diagnosis of rectal lesions with pathologic correlation. J Ultrasound Med 2014; 33: $575-583$.

11. Wang Y, Li L, Wang YX, et al. Time-intensity curve parameters in rectal cancer measured using endorectal ultrasonography with sterile coupling gels filling the rectum: correlations with tumor angiogenesis and clinicopathological features. Biomed Res Int 2014; 2014: 587806.

12. Mainenti PP, Cirillo LC, Camera L, et al. Accuracy of single phase contrast enhanced multidetector CT colonography in the preoperative staging of colo-rectal cancer. Eur J Radiol 2006; 60: 453-459.

13. Sinha R, Verma R, Rajesh A, Richards CJ. Diagnostic value of multidetector row $\mathrm{CT}$ in rectal cancer staging: comparison of multiplanar and axial images with histopathology. Clin Radiol 2006; 61: 924-931.

14. Bipat S, Glas AS, Slors FJ, Zwinderman AH, Bossuyt PM, Stoker J. Rectal cancer: local staging and assessment of lymph node involvement with endoluminal US, CT, and MR imaging - a meta-analysis. Radiology 2004; 232: 773783.

15. Iafrate F, Laghi A, Paolantonio P, et al. Preoperative staging of rectal cancer with MR Imaging: correlation with surgical and histopathologic findings. Radiographics 2006; 26: 701-714.

16. Kim JC, Kim HC, Yu CS, et al. Efficacy of 3-dimensional endorectal ultrasonography compared with conventional ultrasonography and computed tomography in preoperative rectal cancer staging. Am J Surg 2006; 192: 89-97.

17. Hünerbein M, Pegios W, Rau B, Vogl TJ, Felix R, Schlag PM. Prospective comparison of endorectal ultrasound, three-dimensional endorectal ultrasound, and endorectal MRI in the preoperative evaluation of rectal tumors. Preliminary results. Surg Endosc 2000; 14: 1005-1009.
18. Glaser F, Layer G, Zuna I, van Kaick G, Schlag P, Herfarth C. Preoperative assessment of perirectal lymph nodes by ultrasound. Chirurg 1990; 61: 587-591.

19. Hildebrandt U, Feifel G. Importance of endoscopic ultrasonography staging for treatment of rectal cancer. Gastrointest Endosc Clin N Am 1995; 5: 843-849.

20. Heneghan JP, Salem RR, Lange RC, Taylor KJ, Hammers LW. Transrectal sonography in staging rectal carcinoma: the role of gray-scale, color-flow, and Doppler imaging analysis. AJR Am J Roentgenol 1997; 169: 1247-1252.

21. Sudakoff GS, Gasparaitis A, Michelassi F, Hurst R, Hoffmann K, Hackworth C. Endorectal color Doppler imaging of primary and recurrent rectal wall tumors: preliminary experience. AJR Am J Roentgenol 1996; 166: 55-61.

22. Scialpi M, Zottele F, Niccolini M, Dalla Palma F. Transvaginal echography in the study of the rectum. A new method with rectal distension with water. Radiol Med 1993; 85: 203-208.

23. Scialpi M, Niccolini M, Zottele F, Dalla Palma F, Scialpi P. A new method for study of the rectum using transvaginal ultrasound with water enema. Abdom Imaging 1996; 21: 342-344.

24. Scialpi M, Rotondo A, Angelelli G. Water enema transvaginal ultrasound for local staging of stenotic rectal carcinoma. Abdom Imaging 1999; 24: 132-136.

25. Badea R, Badea G, Dejica D, Henegar E. The role of transvaginal sonography as compared with endorectal sonography in the evaluation of rectal cancer: preliminary study. Surg Endosc 1991; 5: 89-91.

26. Kwok H, Bissett IP, Hill GL. Preoperative staging of rectal cancer. Int J Colorectal Dis 2000; 15: 9-20.

27. Brown G, Richards CJ, Newcombe RG, et al. Rectal carcinoma: thin-section MR imaging for staging in 28 patients. Radiology 1999; 211: 215-222.

28. Brown G, Richards CJ, Bourne MW, et al. Morphologic predictors of lymph node status in rectal cancer with use of high-spatial-resolution MR imaging with histopathologic comparison. Radiology 2003; 227: 371-377. 